\title{
The elaboration of a manufacturing flow connectivity model, based on Multi Agent System
}

\author{
Lamyae Fahhama ${ }^{1, *}$, Abdellah Zamma ${ }^{1}$, Khalifa Mansouri ${ }^{1}$, and Zayer Elmajid ${ }^{1}$ \\ ${ }^{1}$ Laboratory SSDIA, ENSET Mohammedia, Hassan II University of Casablanca
}

\begin{abstract}
Nowadays, manufacturing industries are operating in a highly complex and unforeseeable economic context. Therefore, they tend, by necessity toward short term inter-firms coordination. Thus, found increasingly located at the intersection of corporate networks, to overcome their inability to quickly meet the needs of clients, facing thereby several complications related to the coordination between heterogeneous entities. Managing the flow of a network, made up of different links became a major challenge. The appeals to decision support tools and simulation are wished all the more to correlate the dynamics of the system and the targeted objectives, to assess the configurations effectiveness. Thus, enhance their performance.

The aim of this paper was to establish a model of the industrial flow connectivity; Afterward, we've detailed a network configuration model based on the multi-agents systems, to study the interactions between all the actors and give a more realistic vision onto manufacturing coordination in the supply chain.
\end{abstract}

\section{Introduction}

At the beginning of the industrial era, the productivity has been for a long time the principle guiding for a company. Today, with the mutation of the competition and the market globalization, companies became aware that a local effort of improvement of their means of production are not any more enough to reach the level of reactivity and flexibility required to meet the needs of demanding customers. Thus, a tight collaboration and an integration of the various actors of the supply chain turn out, essential to face the exacerbated requests to meet client's satisfaction through the deadline, the cost, the quality of service provided, etc.

Hence, to face these stakes, manufacturing companies, broke more and more of their design activities of production and assembly to the inside of their chain, therefore they're brought to search and benefits from the expertise of potential partners. Thereby, having a multisite, multi-trades design, so they can better respond to the needs of the customers. Changing from a one-level structure to a complex multi-level configuration, that is manufacturing network.

In this perspective, the configuration of manufacturing networks constitutes a problematic major industrial, more particularly in a context of heterogeneous levels. The piloting of the dynamics of the flow of production and the assessment of the performance of the logistics chain incorporated is crucial. It is concerned more precisely to the configuration of a new link or a company Ein combines with a level below a manufacturing network, also named the connectivity of flows [1]. This organizational coalition can be characterized by a number of attributes describing the operating system and the control one of the manufacturing company. These attributes allow defining firms profile able to integrate a manufacturing network. Therefore, supply chain coordination (SCC) offers means to understand and analyze a $\mathrm{SC}$ as a set of dependencies both in physical flows, as well as in informational flows. Our contribution approach is to appraise how efficient the process connectivity in supply chain, made up of heterogeneous, but yet compatible profiles of companies can be. Using communicating agent architecture. The objective of this approach is the anticipation and the evaluation of different scenarios and the detection of the less performing configurations.

Our article is organized as follows: The next section presents a literature review in relation to our work is given; the section 3 introduces our model of flow connectivity in the manufacturing supply chain. In sections 4 we formalize the method of coalitions linking adapted to the distributed coordination by a multi-agent approach. Furthermore, we explain the interactions necessary for its implementation. Finally, we conclude on this work.

\section{The modeling and the simulation of flow connectivity in manufacturing networks}

\subsection{The flow connectivity}

In the Supply chain, manufacturer maintains various types of flow. It may be an information flow, relating to supplies or to the design of products, a financial flow, or even a materials flow raw materials and parts of assembly up to finished products. Coordinating the flow between

${ }^{*}$ Corresponding author: Lamyaefahhama@gmail.com 
two companies then returns, to ensure that the information flows reliably and rapidly between them and that the satisfaction of the customer need be realized in time, with the least cost and the minimum levels of inventory [2-3].

The problems of flow management discussed in the literature are focused as well at the global level between several actors in the SC (inter), that within the activities of one of the actors (intra) [4].

Whereas, researchers were interested in the integration and coordination of the actors of the $\mathrm{SC}$, in order to make the flow more fluid, agile and responsive to the dynamics of the market [4-6]. They had proposed solutions for the management of the $\mathrm{SC}$ often requiring a modification of the organizational, facilitated by the implementation of the new technologies of information and communication. However, the cumbersome and the difficulty of implementing these approaches, often pose problems. In particular, small and medium-sized enterprises, the fact of the rigidity and the limitation of their financial means, cannot always be involved in projects of major restructuration of their organizations [7].

\subsection{The modeling and the simulation of the connectivity}

Works the literature are unanimous on the role of modeling to interpret the reality in order to assimilate it, and criticize it; According to Fischwick, 1997 'The only way to criticize a modeling is to make another by changing the settings' due to the fact that reconfiguration of the parameters in reality is not always possible. Coupled with the simulation, it allows by the conduction of experiments, based on the predetermined model, to study the possible scenarios and evaluate it to deduce the decisions or of findings.

The approach is adopted in several works in order to reduce the complexity of the management of the supply chain and to assess its performance [8]. The models based on communicating agent for the simulation of SC are in vogue, filling thereby the gaps of analytical models to model the chain with all its complexity [9-10]. It's powered in the other hand, by the simulation making possible to manage the stochastic behavior of the Supply Chain before their implementation, and provide a tool to help decision making, by taking into account the dynamics of the system and of the behavior of the different autonomous actors. [11-12].

Some authors, such as [13] have had recourse to the multi-agent systems to resolve the problems of supply chains. Indeed, [14] that had described a framework based on agents for the SCM. Thus, developing a process of negotiation between functional agents. Other work as [15] designs an Information System for the Multimodal Management on the basis of MA, to that is added the thesis of [16] which employment the DAI for the modeling of Meta heuristic, and [17] which he combines the mathematical optimization and the DAI modeling for the development of its system.

It is obvious that, what has been discussed, in the literature around the connectivity of the process of manufacturing networks, established in a supply chain are, either centered on particular industrial problems, or have a qualitative character and thus not reflecting reality. The taking into account of the special features related to planning modes, control of type of production and response to the demand are rarely discussed. Yet they make this system more efficient (reduce delays and stocks and make consistent links). A better model for process connectivity can be proposed, allowing the assets of the performance in an early phase.

Our contribution fallows the same approach of modeling and simulation, to respond to the problem of the configuration of the manufacturing links, within a manufacturing network.

\subsection{The problem description}

In related literature modeling approaches have gained importance in supply chain modeling, there exist vast amount of studies aimed at modeling supply chains under multi-agent setting. However, most of the studies focus on conventional supply chain structures and do not provide a real-world application. In this sense, the notion of 'fit' in an existed supply chain management (connectivity) literature has evolved to achieve greater organizational and operational effectiveness. Raison why the supply chains should be modeled as open and dynamic systems by considering complex adaptive relationships between agents.

In parallel multi-agent system modeling is a suitable approach for conveniently modeling the decision making processes by considering complex relationships. With these ideas gathered, we investigate the issue of supply chain coordination that arises in a supply chain and it is necessary to understand and analyze as a set of dependencies both in physical flows, as well as in informational flows.

A simulation model, which is based on the multi-agent system simulation approach, was considered to help and explore the need to manage these dependencies for a company success, ensure higher productivity through fluent and coherent profiles, and reduce the lead-times and inventory levels among the partners.

The purpose is to address a asses to the coalition making possibility to achieve a state of fit between partners configuration settings and performance indicators, while taking into account the need to achieve a higher service level and customer satisfaction (effectiveness) on the one hand, and being cost-efficient on the other hand (efficiency). 


\section{The proposed model of manufacturing process connectivity}

Unlike the problems of cooperation in the supply chains, existing in the literature, interested in the interactions between the actors, the coordination and sharing of information, our model incorporates the preliminary phases of the establishment of the connection, its establishment and the assessment of its relevance to the achievement of the optimization objectives of the overall chain in any coalition making between partners.

Our model show the different relations between the actors of the supply chain, the configuration of the links between the manufacturing enterprises, of the considered manufacturing network and integrates the algorithm of Coalition [18] to meet the needs of the Existant Link, mainly composed of $\mathrm{E}_{\mathrm{in}}$. Knowing the mode of response to the clients request and the level of capability of partners.

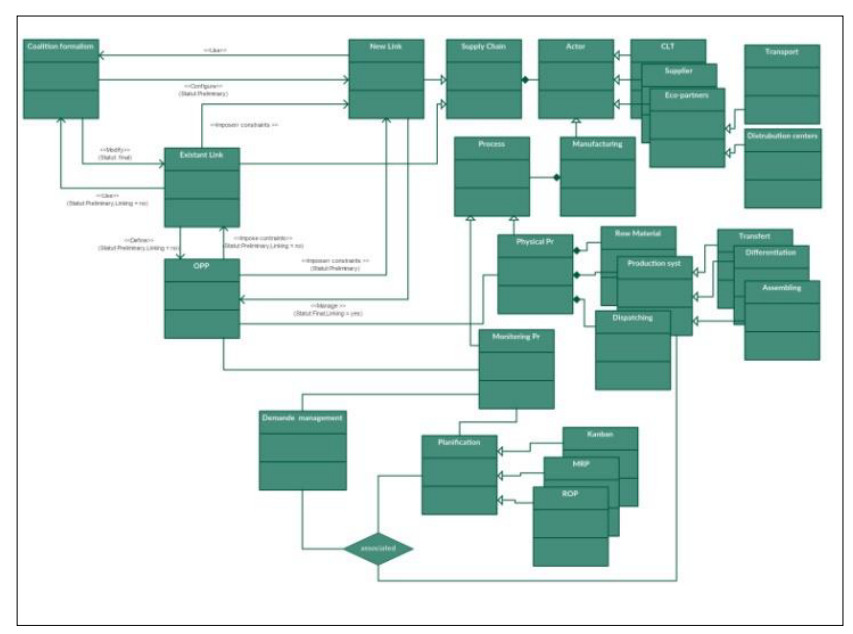

Fig. 1. Class diagram of the connectivity of flow in manufacturing network model.

This modeling will give a better visibility of the problem of connectivity of flows and the configuration of the new links in the same Supply Chain. We briefly present the model proposed of connectivity. We have used the language UML for our modeling and particularly the class diagram (Figure 1).

The connectivity and the need for collaboration, emanates from the position of the process relative to the Order penetration point of command OPP. The characteristics of the products, the type of response to demand, production processes type, planning methods are then identified.

"OPP" is defined from the "Process PH" and the "process of piloting" pre-determined by the "type of response to the client demand" namely MTO, MTS, ATO of the manufacturing company "Manufacturing".

The connectivity of flows under hears the reconfiguration of the chain through the class "formalism of coalition", on which we focus. The class "Existing Link" is the precursor of this configuration, initially composed of an entity of production. In effect, the configuration of the manufacturer's network is considered as a strategic decision requiring a particular intention relative to the performance level wished. The two classes determine and define the vast majority of the constraints to correctly position the types of businesses and thus avoid the design of logistic processes little consistent.

Consequently, the dependence which is established between the Existing Link and the new configured link of the Supply Chain allows à optimized flow stream.

We recall the class "OPP" represents all the constraints related to the configuration of the new link. It is characterized by a Boolean attribute "status", this attribute provides information on the state of the institution or not of a connection. The attribute "status" is a variable which can take two values:

"STATUS = Preliminary", indicating that the coalition referred to the constraints of the $\mathrm{E}_{\mathrm{in}}$ is in it beginning.

"STATUS $=$ final" indicates that there has configured a new link, responding accurately to the expectations of the existing link.

The class "Supply Chain" is a generalization of the classes "Existing Link" and "New Link" to design.

The existing link is a link that is previously available in the Supply Chain. The configuration must satisfy the constraints of this link while satisfying the entire of the technical requirements imposed by the OPP.

The "New link" is a link formed to answer the needs of the Ein available in the network represented by the "Existant Link" in one hand and on the other hand resulted of the treatment of the "Coalition formalism". It will be determined by taking into account the constraints of the mode of response to the demand of the product by the "Existant Link".

The class "Coalition formalism" represents an adapted algorithm of suitable coalition, for the configuration of new link of the SC. The algorithm used has for objectives to train an adequate coalition in the chain, and make a selection based on preference indexes, calculated in reference to determined criteria (Capability of process..); that so to help choosing the best of the heterogeneous entities in terms of means and modes of production.

The "Existant link" exercising its function in interaction with the other actors in the SC. In fact, it's the one which triggers the process of coalition, based on the constraints of the OPP. This class is characterized by a Boolean attribute 'satisfaction' which provides information on the adequacy and the response to its need, by the chosen configuration. The attribute 'satisfaction' is a variable that reflects the state of this satisfaction:

"Satisfaction = yes" which means that all the constraints of this link are met. 
"Satisfaction $=$ no" which means that there is at least a constraint in this link which is not satisfied by the current design.

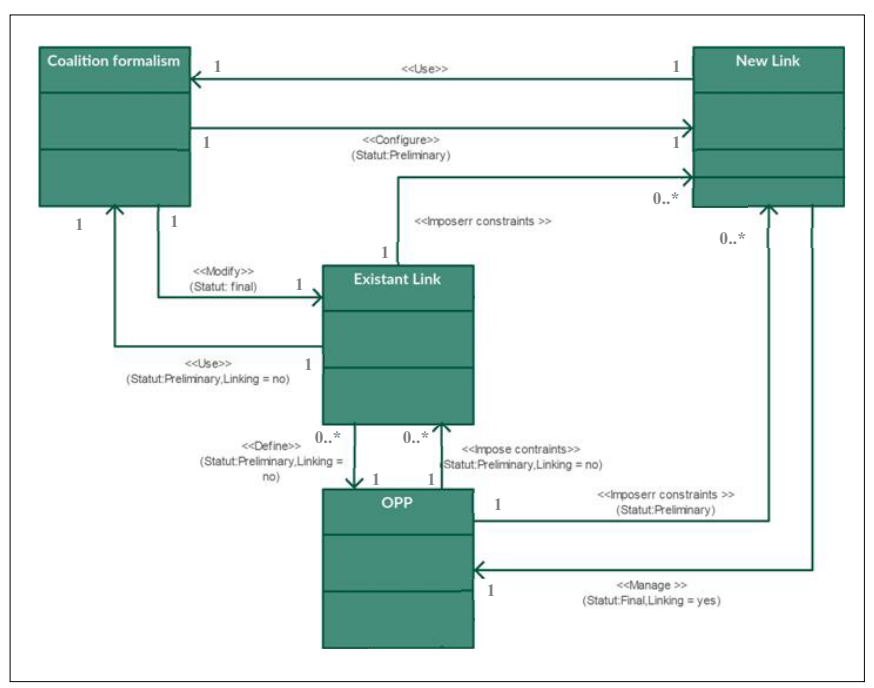

Fig. 2. The class diagram modeling the proposed approach.

The sequence diagram shall present, puts the emphasis on the sequence of messages exchanged between the echelons. It thus, represents the process of coordination in the manufacturing chain between the initiative echelon $E_{i n}$ and its potential partners $E_{1, n}$ in order to establish a synchronization of the physical flows in the case of the establishment of a collaboration between the two or more.

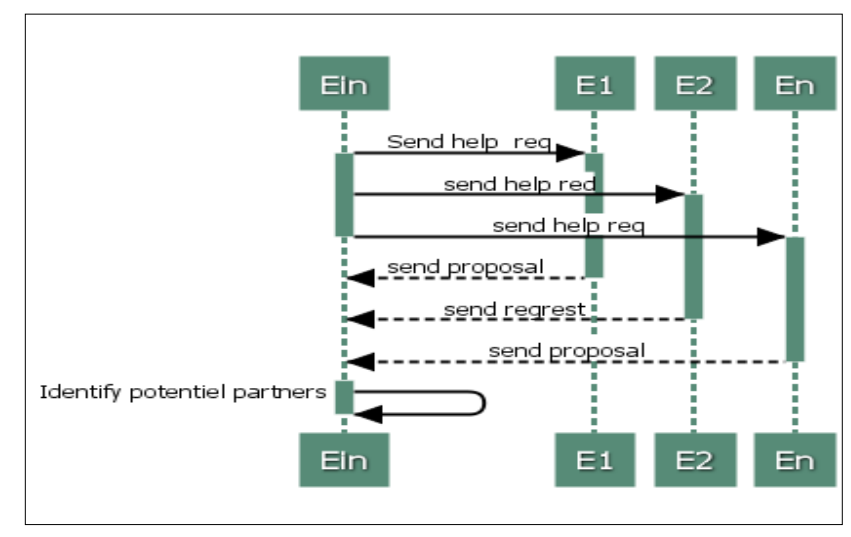

Fig. 3. The sequence diagram for firm collaboration sourcing.

\section{The agents based manufacturing network connectivity}

\subsection{Model main Components}

The analogy and the intersection between the characteristics of the MAS with the structure and the dynamic behavior of decentralized, complex and reconfigurable systems [19] as the connectivity of the manufacturers network, has led us to consider a support for decision-making, by proposing an architecture based on the VOWEL approach [20]. This approach models the concepts of the multi-agent system, resulting from the distributed artificial intelligence and the object oriented modeling, by 4 views to know:

The Agents view: for the internal architecture of agents, the view Environment: to describe the environment in which evolve the agents, an Interaction view: the means by which the agents interact and last but not least, the Organization view: the means used to structure the set of entities.

The Topology group of our architecture includes the group configuration of a new link and the Group Supply Chain; we are interested in the first group which includes the whole of the agents participating in the coalition formation .Composed of agents 'coalition', 'Configuration', 'Decision', 'AIC' and Agent 'Existing Link'. The table 1 below contains the agents of the proposed architecture centered on the configuration of the manufacturing network, gives the name, the type and the role of each:

Table 1. Agent identification by type and role.

\begin{tabular}{|l|l|l|}
\hline \multicolumn{1}{|c|}{ Agent Name } & \multicolumn{1}{|c|}{ Type } & \multicolumn{1}{c|}{ Role } \\
\hline $\begin{array}{l}\text { Existant Link } \\
\text { Agent }\end{array}$ & Réactif & $\begin{array}{l}\text { Collect information of echelons, } \\
\text { Introduce the connectivity, define the } \\
\text { objectives and has a knowledge of the } \\
\text { constraints of the OPP, }\end{array}$ \\
\hline Configuration & Cognitif & $\begin{array}{l}\text { Propose adapted new links based on } \\
\text { the information received from the } \\
\text { agent Coalition. Receives the } \\
\text { improvements of the agent Decision } \\
\text { and transmits the configuration to the } \\
\text { agent AIC, }\end{array}$ \\
\hline Coalition Agent & Cognitif & $\begin{array}{l}\text { Analyze the objectives of the existing } \\
\text { link and the constraints of the OPP and } \\
\text { handles the information received from } \\
\text { all other agents to communicate them } \\
\text { to the Configuration agent, }\end{array}$ \\
\hline Decision Agent & Cognitif & $\begin{array}{l}\text { Analyze the configuration predefined } \\
\text { by the Configuration agent and } \\
\text { decide of the preservation or not of the } \\
\text { connection, }\end{array}$ \\
\hline AIC Agent & Réactif & $\begin{array}{l}\text { Ensure communication between } \\
\text { agents. }\end{array}$ \\
\hline
\end{tabular}

To those we add, the group supply chain, through which we model the entities of the SC by agents. To know the agents: Agent 'supplier', Agent 'client', Agent 'Eco-partner', Agent 'manufacturing'.

Each agent has a set of rules and behavior that it implements to succeed this configuration approach of the manufacturing network and this in order to achieve the level of the capability required and an efficient configuration. In what follows, we present our model organization and the interactions of the agents, modeling their dynamic behavior [21].

\subsection{The Proposed Agent based Model}




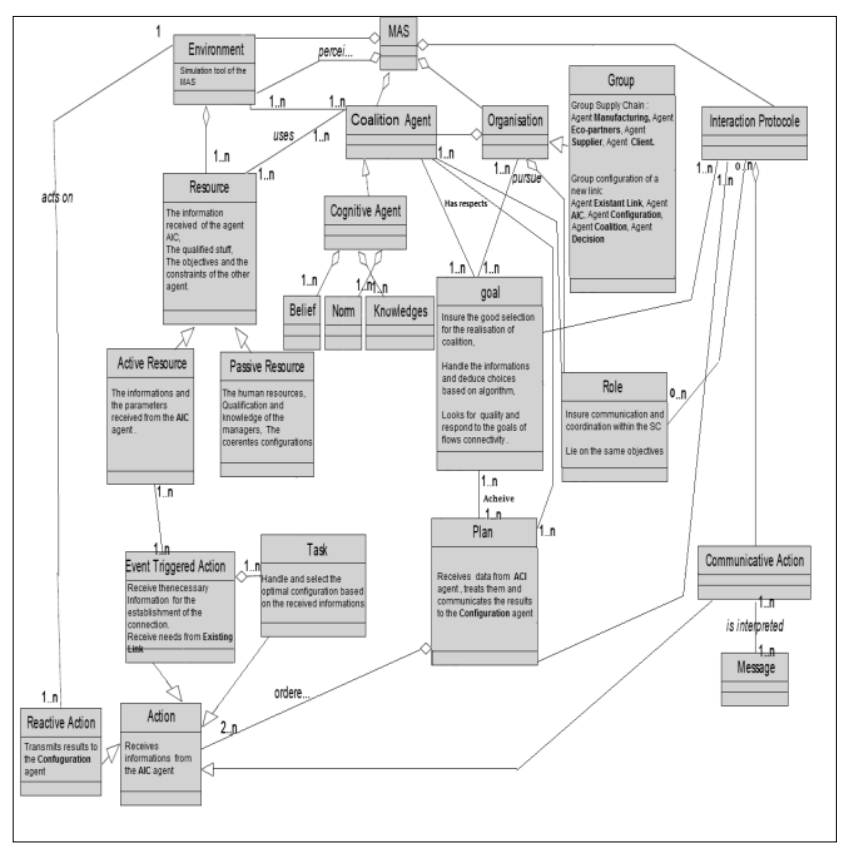

Fig. 4. The global multi-agent model identifies different views of the AEIO approach.

The configuration of the manufacturing network is formalized by the sequence diagram bellow using AUML (Figure 5). The agent 'AIC' receives the information from the agent 'Existant Link' and under hears a communication with the other agents of the Supply Chain. The information comprising the formulation of the specific needs, constraints and objectives including the selection of potential partners, are sent to the agent 'Coalition', where they are treated. The proposition of a selected partner $E_{1, n}$ is sent to the agent 'Configuration' responsible of the configuration of a new link.

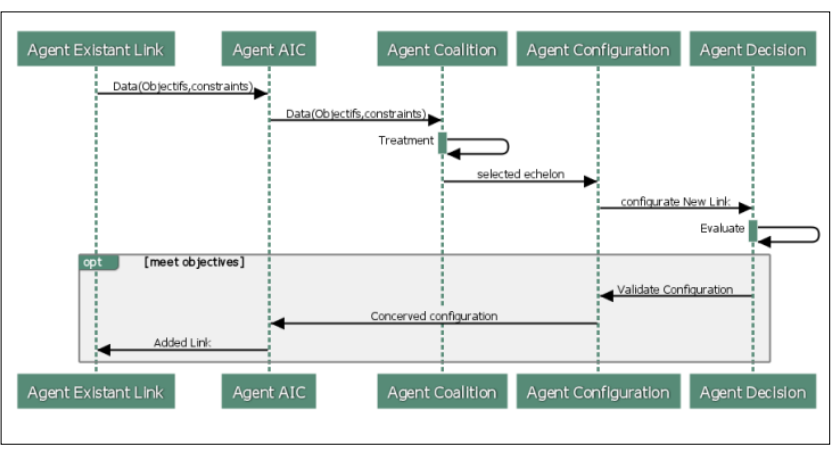

Fig. 5. The sequential diagram of interaction and communication between agents.

The agent 'Configuration', ensures according to the information received from the agent 'Coalition' the reconfiguration of the manufacturing network, the relevance and validity of this new link will be assessed at the level of the agent 'Decision', who can either validate the configuration of the new link, as it may not.

The confirmed configuration will be sent to the agent 'AIC' to be broadcast to the agents of the chain.
This process ended, when the agent 'Decision' validates a configuration responding to constraints (objectives, the adequacy to the processes homogeneity, capability of the SC). The echelon added will be considered as an 'Existing Link'.

\section{Summary and outlook}

The work presented in this paper is related to the field of integrated engineering, we focused on flow connectivity in the manufacturing network. Managing this heterogeneous form of configurations is mainly based on an information sharing and matching activities between the various links.

In a first step, a systematic literature review has been considered includes different topics related to SCM, its modeling and its simulation in order to explore all types of models, used by several researchers. The aim of this step was to be inspired later, while developing our model presented in the second step.

Thus, we've presented an agent based model of the flow connectivity, the framework able to analyze quality, and effectiveness of configured links and their effects on performance. In addition, we've illustrated the whole interactions between the various agents. The employment of the MAS in linking process addresses many issues where future research could yield interesting results.

\section{Acknowledgement}

The authors gratefully acknowledge financial support from The National Center for Scientific and Technical Research (CNRST), Morocco.

\section{References}

1. M. Essaid, "Modélisation et simulation de la connectivité des flux logistiques dans les réseaux manufacturiers," p. 207,(2013)

2. N. V. K. Jasti and R. Kodali, Int. J. Prod. Res., no. August, pp. 1-19, (2014)

3. A. Kaddoussi, "Optimisation des flux logistiques: vers une gestion avancée de la situation de crise," Trait. du Signal, (2012)

4. D. Anane, S. Pinson, and S. Aknine, "Les Approches Agents Pour La Coordination D'Activités Dans Les Chaînes Logistiques," Cah. Du Lamsade, $\underline{(2009)}$

5. T. Vadvári and P. Várlaki, "Identification of Supply Chains Based on Input-Output Data," Period. Polytech. Transp. Eng., vol. 43, no. 3, pp. 162-167, (2015). DOI: $\underline{10.3311 / \text { PPtr.7931. }}$ 
6. X. Zhang, "Analysis for Scale-Free Network Characteristics of Logistics Distribution Network," J. Serv. Sci. Manag., vol. 7, no. 3, pp. 189-195, (2014).DIO: $10.3311 /$ PPtr.7931

7. F. Demoly, X. T. Yan, B. Eynard, S. Gomes and D. Kiritsis, "Integrated product relationships management: a model to enable concurrent product design and assembly sequence planning", J.Eng.Des. , Vol. 23, no 7, pp. 544561, (2012). DIO: $10.1080 / 09544828.2011 .629317$

8. Y. Chu, F. You, J. M. Wassick, and A. Agarwal, "Simulation-based optimization framework for multiechelon inventory systems under uncertainty," Comput. Chem. Eng., vol. 73, no. 2015, pp. 1-16, (2015). DIO: 10.1016/j.compchemeng.2014.10.008

9. P. Jain , D. Dahiya, "An Architecture of a Multi Agent Enterprise Knowledge Management System Based on Service Oriented Architecture", IJCSI, Vol. 9, Issue 2, No 3,(2012).

10. H. Nfaoui, Y. Ozrout, "SCM, AUML : un modèle d'agents pour la simulation proactive et l'aide à la décision dans le Supply Chain", Conférence Majestic, MAnifestation des JEunes Chercheurs en Sciences et Technologies de l'Information et de la Communication. 22-24, Lorient France, (2006)

11. J. Costas, B. Ponte, D. de la Fuente, R. Pino, J. Puche, Applying Goldratt's theory of constraints to reduce the bullwhip effect through agent-based modeling, Exp. Syst. Appl. 42 (2015) 2049-2060.

12. V. Kumar, S. Srinivasan, "A Review of Supply Chain Management using Multi-Agent System", IJCSI, Vol. 7, Issue 5, (2010).

13. W.E. Walsh, M.P. Wellman, "Modeling Supply Chain formation in multiagent systems", In: Agent Mediated Electronic Commerce (IJCAI Workshop), 94101, (1999).

14. Y. Chen, Y. Peng, T. Finin, Y. Labrou, S. Cost, B. Chu, J. Yao, R. Sun and B Wilhelm, "A negotiation based multi agent system for Supply Chain", In: Working Notes of the Agents '99 Workshop on Agents for Electronic Commerce and Managing the Internet-Enabled Supply Chain, Seattle, WA, April (1999).

15. M. A. Kammoun, Conception d'un système d'information pour l'aide au déplacement multimodal : Une approche multi-agents pour la recherche et la composition des itinéraires en ligne. Lille: Ecole Centrale de Lille, (2007).

16. Y. Chu, F. You, J. M. Wassick, and A. Agarwal, "Simulation-based optimization framework for multiechelon inventory systems under uncertainty," Comput. Chem. Eng., vol. 73, no. 2015, pp. 1-16, (2015), DIO: 10.1016/j.compchemeng.2014.10.008
17. M. Sghaier, Combinaison des techniques d'optimisation et de l'intelligence artificielle distribuée pour la mise en place d'un système de covoiturage dynamique. Ecole Centrale de Lille, (2011).

18. A. Mortazavi, A. Arshadi Khamseh, and P. Azimi, "Designing of an intelligent self-adaptive model for supply chain ordering management system," Eng. Appl. Artif. Intell., vol. 37, no. March, pp. 207-220, (2015). DIO:10.1016/j.engappai.2014.09.004

19. W. Wang, W. Fu, H. Zhang, and Y. Wang, "Hybrid modeling and simulation of automotive supply chain network," vol. 6, no. 9, pp. 1598-1605, (2013). ISSN: 20407459

20. M.Shaiq, R. Shaikh and A.Ahmed, Conflict Resolutions in Supply Chain Collaboration a Perspective of Supply Chain Managers, Journal of Business Strategies, Vol. 9, No.1, pp. 17-30, (2015)

21. A. Seco and C. Vieira, "A multi-agent supply chain simulation analysis through a statistical mixed model," Procedia Technol., vol. 16, pp. 163-171, (2014). DIO:10.1016/j.protcy.2014.10.079. 